\title{
IDENTIFICAÇÃO DE CERRADO RUPESTRE POR MEIO DE IMAGENS MULTITEMPORAIS DO LANDSAT: PROPOSTA METODOLÓGICA
}

\section{Identification of Rupestrian Cerrado using multitemporal Landsat images: methodological approach}

\author{
Erika Regina Prado Nascimento \\ Universidade de Brasília \\ Brasília/DF - Brasil \\ erikaregina@unb.br \\ Edson Eyji Sano \\ Embrapa Cerrados \\ Brasília/DF - Brasil \\ sano@cpac.embrapa.br
}

Artigo recebido para publicação em 29/01/2010 e aceito para publicação em 06/04/2010

RESUMO: $\quad$ O Cerrado Rupestre corresponde a uma formação savânica do bioma Cerrado, ocorre em relevos acidentados e em meio a afloramentos rochosos, apresenta elevada biodiversidade e várias espécies endêmicas e funciona como barreira para a expansão agrícola. No estado de Goiás, ocorrências expressivas dessa fitofisionomia são encontradas no Parque Nacional da Chapada dos Veadeiros (PNCV). O objetivo deste estudo foi desenvolver uma nova abordagem metodológica para discriminar Cerrado Rupestre do PNCV com base em imagens multitemporais do satélite Landsat. Sete cenas do referido satélite foram convertidas para reflectância de superficie terrestre com suporte do algoritmo de correção atmosférica denominado FLAASH (Fast Line-of-Sight Atmospheric Analysis of Spectral Hypercubes). Em seguida, os valores de reflectância de cada cena foram somados e as imagens resultantes foram processadas por meio da técnica de segmentação de imagens por crescimento de regiões. Os segmentos foram exportados para o formato shape e os poligonos correspondentes ao Cerrado Rupestre foram identificados por meio de análise visual na tela do computador da composição colorida falsa-cor das bandas 3, 4 e 5. Foram mapeados 24.451 hectares de Cerrado Rupestre, o que corresponde a $37 \%$ da área do parque. A exatidão global do mapeamento foi de $85 \%$. Como continuação desta linha de pesquisa, recomenda-se a inclusão de um número maior de cenas, principalmente da estação chuvosa, a integração dos dados de sensoriamento remoto com modelos digitais de elevação e a análise sinergística entre os sensores ETM+e TM do Landsat com calibração cruzada.

Palavras chave: Sensoriamento Remoto. Correção Atmosférica. Reflectância. Segmentação de Imagens.

ABSTRACT: The Rupestrian Cerrado (Cerrado Rupestre) corresponds to a shrub-like vegetation of the Brazilian tropical savanna biome, occurs mainly over hilly topography and rocky outcrops, presents high biodiversity and several endemic species, and acts as barrier for agricultural expansion. In the State of Goias, large occurrences of this type of vegetation are found in the Chapada dos Veadeiros National Park (PNCV - Parque Nacional da Chapada dos Veadeiros). Rupestrian Cerrado mapping using remotely 
Identificação de Cerrado Rupestre por Meio de Imagens Multitemporais do Landsat: Proposta Metodológica Erika Regina Prado Nascimento, Edson Eyji Sano

sensed data is difficult because of the spectral confusion with other phytophysiognomies, especially with Dry Forest in dry season images and with Cerrado strictu sensu in wet season images. The goal of this study was to develop a new approach to discriminate Rupestrian Cerrado based on multitemporal Landsat satellite images. The study area was the PNCV. Seven Landsat scenes were converted into surface reflectances with support of FLAASH (Fast Line-of-Sight Atmospheric Analysis of Spectral Hypercubes) atmospheric correction algorithm. The reflectances from each ETM+ band were summed and the resulting images were processed through the image segmentation technique by growing region. The segments were exported into shapefile format and the polygons related to the Rupestrian Cerrado were identified by visual analysis of the false color composite of bands 3, 4 and 5 in the computer screen. We found 24,451 hectares of Rupestrian Cerrado, which corresponds to 37\% of total area of the park. The global accuracy of the mapping was $85 \%$. As ongoing research, we recommend the inclusion of a higher number of scenes, mainly from wet season, the data integration of satellite images with digital elevation models, and the synergistic analysis of cross-calibrated Landsat ETM+ and TM data sets.

Keywords: remote Sensing, Atmospheric Correction. Reflectance. Image Segmentation.

\section{INTRODUÇÃO}

O Cerrado Rupestre corresponde a uma das fitofisionomias representativas do bioma Cerrado e é típico de ambientes rupestres, os quais englobam cerca de $7 \%$ da região de domínio do referido bioma (REATTO et al., 2008). O Cerrado Rupestre apresenta grau relativamente elevado de conservação, pois ocorrem sob condições topográficas e edáficas peculiares, isto é, elevadas altitudes e em solos rasos e com elevado número de afloramentos de rochas de quartzito e arenito. Consequentemente, as suas áreas de ocorrência atuam como importantes barreiras para a expansão agrícola no Cerrado e para a conservação da vida silvestre do bioma.

O Parque Nacional da Chapada dos Veadeiros (PNCV), localizado na porção nordeste de Goiás, constitui-se em uma das áreas mais expressivas em termos de ocorrência de Cerrado Rupestre no estado.

Estudos anteriores têm mostrado certa dificuldade em discriminar fitofisionomias do Cerrado por meio de imagens ópticas. Por exemplo, Sano et al. (2005) analisaram o desempenho dos índices de vegetação por diferença normalizada (NDVI) e realçado (EVI), derivados de imagens do satélite Landsat para discriminar classes de cobertura vegetal presentes no Parque Nacional de Brasília. Somente dois grupos de cobertura vegetal puderam ser separados: um, formado pela Mata de Galeria e o outro, formado pelas formações campestres e savânicas. Lima et al. (2009) também mencionaram confusão espectral entre Cerrado Rupestre, Cerrado sentido restrito, Mata Seca semidecídua e Mata Seca decídua em imagens do Landsat da Área de Proteção Ambiental Pouso Alto, Goiás. Tais dificuldades estão associadas com a elevada sazonalidade climática e com a transição gradual entre as fitofisionomias do Cerrado.

Alguns autores têm buscado alternativas para obter discriminação espectral de fitofisionomias do Cerrado com acurácia maior. Liesenberg et al. (2007) analisaram dados do MISR (Multi-Angle Imaging SpectroRradiometer), a bordo da plataforma Terra e com capacidade de imageamento em nove ângulos distintos de incidência, para discriminar cinco fitofisionomias presentes nos limites dos estados de Minas Gerais e da Bahia. Resultados indicaram que imageamentos off-nadir possuem elevado potencial para a obtenção de classificações vegetais mais acuradas. Ratana et al. (2005) demonstraram, por meio de análise de dados multitemporais do MODIS

Sociedade \& Natureza, Uberlândia, 22 (1): 93-106, abr. 2010 
(Moderate Resolution Imaging Spectroradiometer), que os índices de vegetação acumulados em um ano podem melhorar a capacidade de discriminar fitofisionomias do Cerrado. As formações florestais tendem a apresentarem índices acumulados maiores que as formações savânicas e campestres.

Infelizmente, ambos os dados, MISR e MODIS, apresentam resolução espacial grosseira - aproximadamente 1 quilômetro para o MISR e 250 metros para o MODIS - , o que inviabiliza o mapeamento de cobertura vegetal em escalas detalhadas (1:100.000 ou superior). Nesse sentido, novas abordagens metodológicas baseadas em imagens com resolução espacial decimétrica (família do Landsat ou similar) ainda precisam ser desenvolvidas e testadas no sentido de se obter discriminação melhorada de fitofisionomias do Cerrado.
Considerando-se a necessidade de se realizar pesquisas que se referem às técnicas de mapeamento de Cerrado Rupestre, foi proposta uma nova metodologia para o mapeamento dessa fitofisionomia. O estudo teve como objetivo principal apresentar uma nova abordagem metodológica para discriminar Cerrado Rupestre do PNCV com base em imagens multitemporais do satélite Landsat.

\section{MATERIAIS E MÉTODOS}

O PNCV, com uma extensão de 65.514 hectares, está localizado no segmento nordeste do estado de Goiás, entre as latitudes $13^{\circ} 51^{\prime} \mathrm{S}$ e $14^{\circ} 10^{\prime} \mathrm{S}$ e entre as longitudes $47^{\circ} 25^{\prime} \mathrm{e} 47^{\circ} 42^{\prime} \mathrm{W}$. Abrange os municípios de Alto Paraíso de Goiás, Cavalcante e Terezina de Goiás (ao norte) e Colinas do Sul (a oeste) (FIGURA 1).

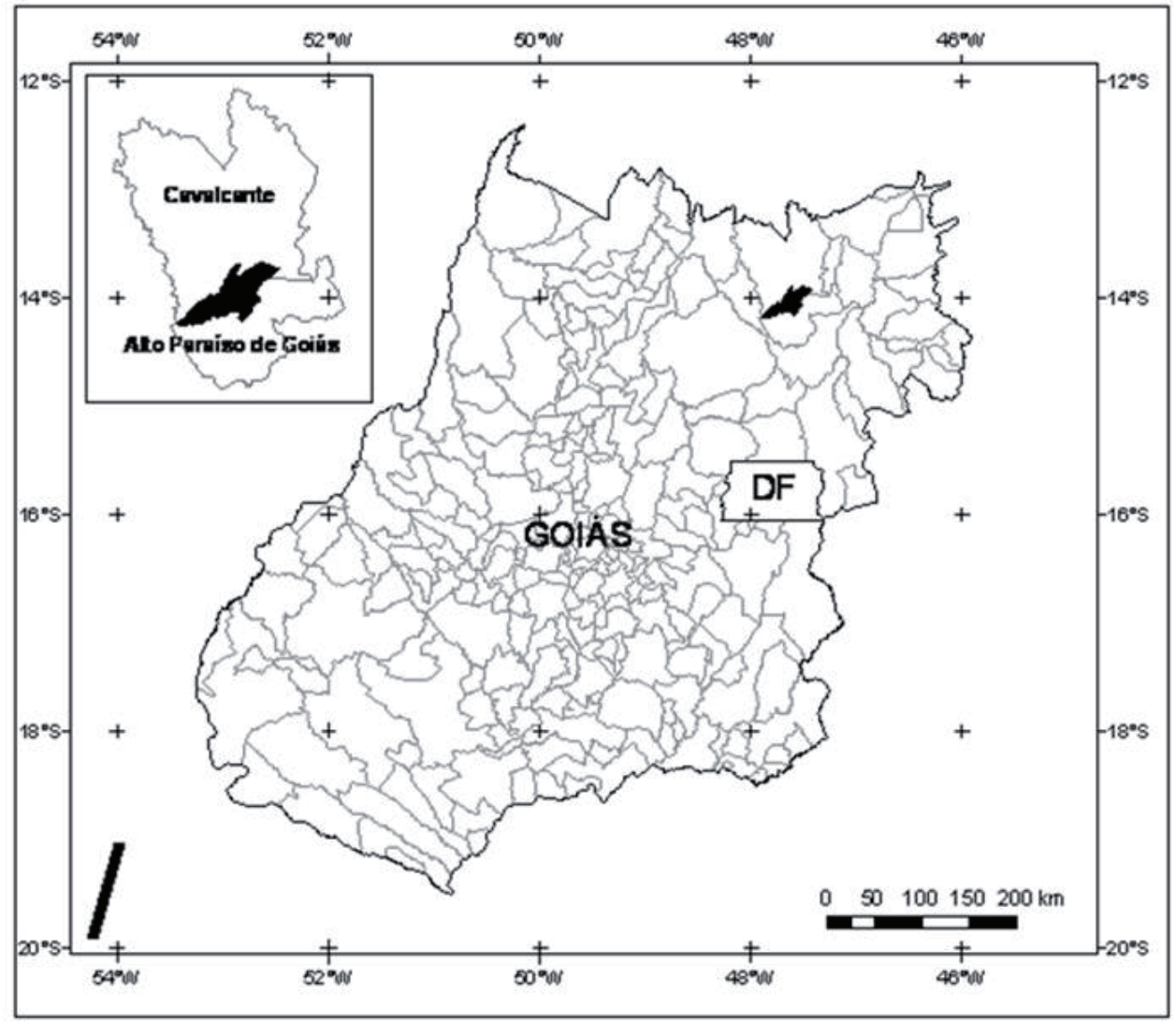

FIGURA 1: Localização da área de estudo no estado de Goiás. 
Identificação de Cerrado Rupestre por Meio de Imagens Multitemporais do Landsat: Proposta Metodológica Erika Regina Prado Nascimento, Edson Eyji Sano

Foram obtidas da página eletrônica do Instituto Nacional de Pesquisas Espaciais (http:// www.dgi.inpe.br/CDSR), sete cenas do Landsat $\mathrm{ETM}+$ de 2001 e 2002, correspondentes a orbita 221 e ponto 70 (TABELA 1). As cenas foram adquiridas nos meses de junho, julho, setembro e outubro. De acordo com a série histórica (1984 - 2003) de dados Landsat do Cerrado, o período de maio a outubro é o que apresenta maior probabilidade - superior a $40 \%$ - de se obter imagens Landsat desse bioma com menos de $10 \%$ de cobertura de nuvens (SANO et al., 2007). Estimativas visuais de cobertura de nuvens indicaram uma porcentagem de cobertura de nuvens sobre a área de estudo de até $20 \%$ em duas cenas. Nesse estudo, preferiu-se não utilizar outras imagens do Landsat TM, disponíveis na área de estudo e sem cobertura de nuvens, por causa das dificuldades em convertê-los em reflectância de superfície. Testes detalhados que foram conduzidos em uma cena de 2008 não mostraram resultados satisfatórios, provavelmente por causa de valores de ganho e offset que não estão mais confiáveis, de acordo com Vicente-Serrano et al. (2008).

TABELA 1: Características das imagens Landsat ETM+ do Parque Nacional da Chapada dos Veadeiros, utilizadas para a discriminação de Cerrado Rupestre.

\begin{tabular}{ccc}
\hline Órbita/Ponto & Data de Passagem & $\begin{array}{c}\text { Estimativa visual de } \\
\text { cobertura de nuvens sobre a } \\
\text { área de estudo }\end{array}$ \\
\hline & 02 de junho de 2001 & $0 \%$ \\
20 de julho de 2001 & $0 \%$ \\
$221 / 70$ & 06 de setembro de 2001 & $0 \%$ \\
& 22 de setembro de 2001 & $20 \%$ \\
& 05 de junho de 2002 & $0 \%$ \\
& 23 de julho de 2002 & $20 \%$ \\
& 11 de outubro de 2002 & $10 \%$ \\
\hline
\end{tabular}

Foi utilizado também um mosaico de imagens ortorretificadas do parque com equalização de histogramas e com 1 metro de resolução espacial, composto de 12 imagens Ikonos dos meses de junho e julho de 2003 e três imagens QuickBird de 11 de junho de 2003. O mosaico foi georreferenciado para o sistema de projeção UTM (Universal Transversa de Mercator), datum WGS84 e zona 23S. As imagens do QuickBird, com 60 centímetros de resolução, foram reamostradas para 1 metro. A ortorretificação foi feita a partir de um modelo digital de elevação que foi gerado com base em imagens do Terra/ ASTER. Todos os processamentos efetuados para a geração desse mosaico foram conduzidos pela Geoambiente Sensoriamento Remoto ${ }^{\mathrm{TM}}$ (GEOAMBIENTE, 2005).

Cada imagem original do Landsat ETM+ foi inicialmente convertida em radiância no topo da atmosfera por meio do aplicativo ENVI 4.5. Os principais dados exigidos na etapa de conversão foram: data e horário da passagem do satélite; coordenadas centrais da cena; ângulo de elevação solar; altitude média do terreno; valores de ganho e offset; e condições da atmosfera no momento da passagem do satélite (visibilidade; concentração de gases e aerossóis). Nesse trabalho, esse procedimento foi feito banda a banda (total: seis bandas) para as 
sete cenas utilizadas no estudo. Os parâmetros de ganho e offset do satélite Landsat foram obtidos no seguinte endereço eletrônico: http://landsat.usgs. gov/science_L7_cpf.php.

Por causa de especificidades em termos de unidades dos parâmetros de entrada do FLAASH (algoritmo de correção atmosférica disponível no ENVI), as unidades de radiância foram convertidas de $\mathrm{W} /\left(\mathrm{m}^{2}\right.$. sr. $\left.\mu \mathrm{m}\right)$ para $\mu \mathrm{W} /\left(\mathrm{cm}^{2}\right.$.sr.nm). Em termos práticos, basta dividir o valor de radiância resultante de cada banda por 10. As imagens resultantes foram ainda transformadas do formato BSQ (bandsequential) para o formato BIL (band-interleavedby-line). Esses dois formatos representam maneiras diferentes de armazenar imagens digitais com múltiplas bandas. $\mathrm{O}$ primeiro armazena os valores digitais de todos os pixels de uma banda em seqüência, enquanto o segundo armazena todos os valores digitais do primeiro pixel de $\mathrm{n}$ bandas em sequência.

Em seguida, as imagens de radiância no topo da atmosfera foram convertidas para reflectância na superfície terrestre por meio do algoritmo denominado FLAASH disponível no ENVI 4.5. Para cada cena, foram inseridas as informações referentes à altitude nominal do sensor, data e hora do vôo e coordenadas centrais de cada cena. A elevação média do terreno no parque foi assumida como sendo de $1,5 \mathrm{~km}$. O modelo atmosférico usado foi o tropical e o modelo de aerosol foi o rural. $O$ fator multiplicador da coluna d'água utilizado foi 1 e a visibilidade inicial adotada foi de $100 \mathrm{~km}$. Para avaliar o desempenho dessa conversão, valores de reflectância de Mata de Galeria do PNCV foram comparados com os do Parque Nacional de Brasília. Os dados de reflectância deste último parque foram obtidos de uma forma bastante acurada por meio do modelo de transferência radiativa $6 \mathrm{~S}$ e suporte de dados espectroradiométricos de campo, obtidos concomitantemente com a passagem do satélite (órbita: 221; ponto: 71), por meio do espectrorradiômetro FieldSpec da Universidade do Arizona, Estados Unidos (dados não publicados).

As imagens convertidas em reflectância foram georreferenciadas com suporte do aplicativo
Erdas Imagine 9.1, extensão IMAGINE AutoSync. O modelo geométrico para correção que foi utilizado correspondeu ao tipo affine projection model (APM), também conhecido como equação de projeção paralela. Esse modelo permite a extração precisa de informações tridimensionais do terreno sem o conhecimento dos parâmetros geométricos do sensor e órbita do satélite (CERQUEIRA et al., 2003). O valor de erro quadrático médio (RMS) adotado foi de 0,5 pixels. O georreferenciamento foi feito com base na imagem ortorretificada (geocover) do Landsat ETM+ do PNCV de 05 de agosto de 2001, disponível no endereço eletrônico da Universidade de Maryland (http://glcfapp.umiacs umd.edu:8080/esdi/index. jsp).

$$
\rho_{a}=\sum_{i=1}^{n} \rho_{i}
$$

onde $\mathrm{r}_{a}=$ reflectância acumulada de uma determinada banda; $\mathrm{r} i=$ reflectância de uma determinada cena e determinada banda.

As imagens com reflectância acumulada das seis bandas do Landsat foram segmentadas pela técnica de crescimento de regiões. Após vários testes com diferentes valores, foram utilizados os seguintes limiares de similaridade e tamanho de área: $50 \mathrm{e}$ 30 , respectivamente. Os segmentos gerados foram exportados no formato shape. Foram gerados 4.046 polígonos, variando de 1,4 a 2.735 hectares.

Com o objetivo de identificar um maior número possível de ocorrências de Cerrado Rupestre no parque para subsidiar a interpretação de imagens, um trabalho de campo foi conduzido no PNCV no período de 07 a 08 de maio de 2009. Nesse trabalho de campo, os pontos visitados foram identificados por meio de coordenadas geográficas obtidas por um receptor GPS de navegação. A identificação da fitofisionomia foi realizada por meio de inspeção visual do local. Um total de 26 pontos amostrais foi visitado nessa campanha. As fitofisionomias identificadas foram: Campo Limpo (seis pontos); Campo Limpo Úmido (três pontos); Campo Sujo (cinco pontos); Cerrado Ralo (dois pontos); Cerrado 
Rupestre (sete pontos); Cerrado Típico (dois pontos); e Vereda (um ponto). Esse número relativamente pequeno de pontos amostrados foi conseqüência da escassez de vias de acesso no interior do parque. Na FIGURA 2, são mostrados os padrões espectrais representativos de Cerrado Rupestre na composição colorida. Percebe-se que tal fitofisionomia apresenta uma coloração esverdeada ou verde-escura, esta última provavelmente devido ao sombreamento de relevo (o Cerrado Rupestre ocorre tipicamente em relevos acidentados). Em termos de textura, o predomínio é de uma textura intermediária a rugosa.

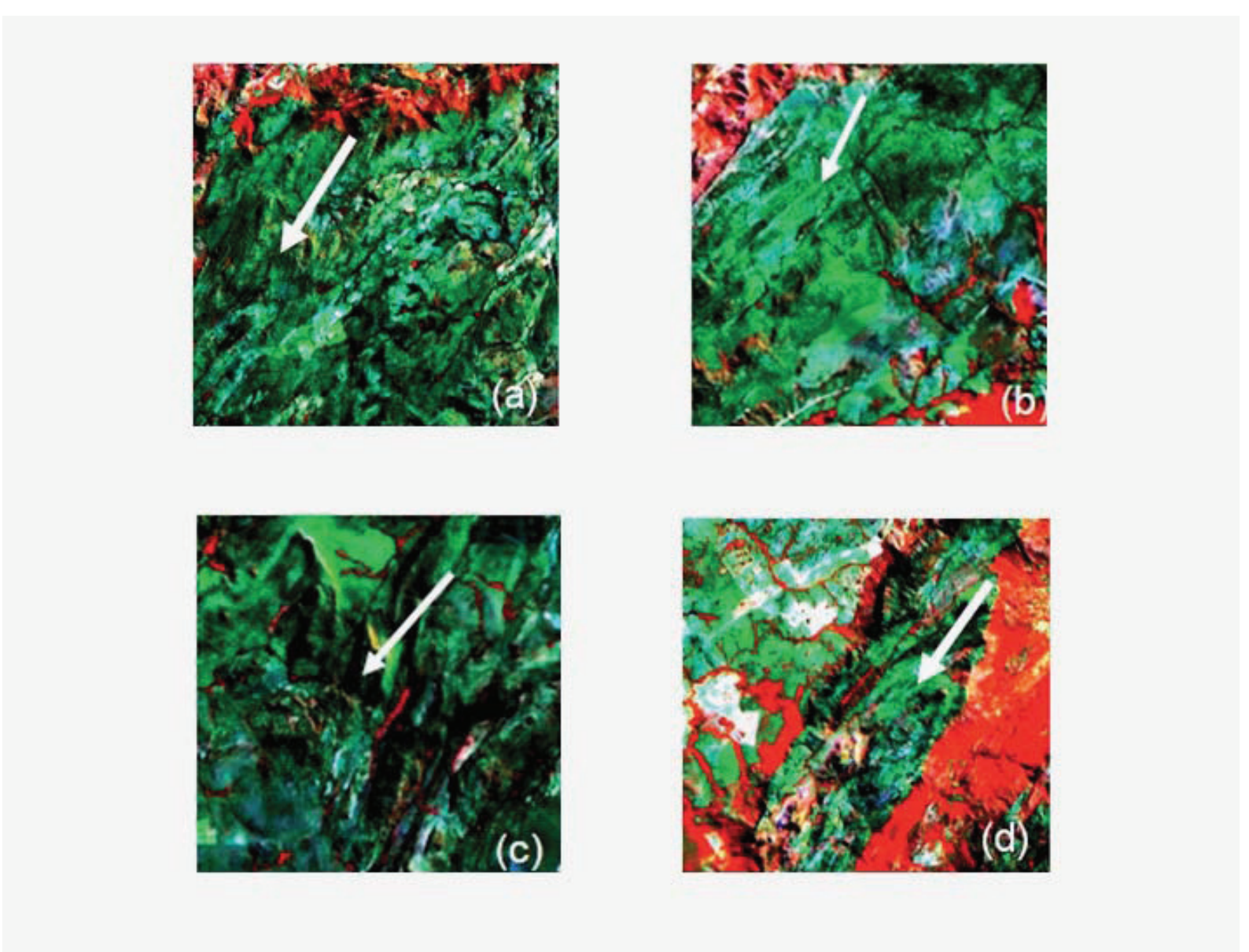

FIGURA 2: Representações típicas de padrões espectral(seria necessário mostrar os espectros) e textural de Cerrado Rupestre no Parque Nacional da Chapada dos Veadeiros e na composição colorida RGB/453 de imagens do Landsat $\mathrm{ETM}+$ convertidas em reflectância acumulada. O que significa a,b,c e d.

Com base nessa chave de interpretação, os segmentos correspondentes a Cerrado Rupestre foram identificados visualmente na tela de computador. Para isso, os segmentos no formato vetorial foram sobrepostos na composição colorida RGB/453. A escala de mapeamento na tela foi de 1:40.000.

Conforme mencionado anteriormente, a validação da interpretação de imagens Landsat foi realizada por meio do mosaico de imagens Ikonos e QuickBird. Foi gerada uma grade regular de pontos com espaçamento de 30 segundos ( 925 metros), num total de 781 pontos. Validação refere-se a um processo de avaliação, por meio de um método independente, da qualidade de um produto gerado (JUSTICE et al., 2000). Nesse contexto, mapas ou interpretações produzidos por sensores ETM+ ou similares, com resolução espacial decimétrica, 
podem ser validados, por exemplo, por meio de campanhas de campo, fotografias aéreas ou imagens com resolução espacial mais fina (MORISETTE et al., 2003). Por meio de inspeção visual do mosaico de imagens do Ikonos e QuickBird, cada ponto foi identificado como "Cerrado Rupestre" ou "Não Rupestre", a partir de uma inspeção visual. Do total de 781 pontos, 79 pontos foram desconsiderados por apresentar grau de incerteza baixo na referida identificação.

Esse número de pontos é aproximadamente 3,4 vezes superior ao número mínimo de pontos $(N)$, o qual pode ser determinado conforme a relação indicada por Jensen (1996):

$$
N=\frac{4(p \gamma q)}{E^{2}}
$$

onde $p=$ percentual de exatidão esperado; $q$ $=1-p ;$ e $E=$ erro permissível. Para um percentual de exatidão de $85 \%$ e um erro admissível de $5 \%$, o número mínimo de pontos é igual a 204.

Esses pontos discriminados foram sobrepostos ao mapa de ocorrência de Cerrado Rupestre do PNCV para se calcular a matriz de confusão do mapeamento final. A matriz de confusão estima o erro global do mapeamento para cada categoria (BRITES et al., 1996; CAMPBELL, 2007).

\section{RESULTADOS E DISCUSSÃO}

Para avaliar o desempenho da conversão de valores digitais originais das imagens Landsat ETM+ para reflectância de superfície, foram extraídas 10 regiões de interesse (ROI) sobre a Mata de Galeria, um alvo com assinatura espectral bem definida e relativamente estável no tempo, isto é, com baixa sensibilidade às variações relativas à sazonalidade climática. Os ROIs correspondentes à Mata de Galeria estiveram distribuídos por toda a área coberta pela cena 221/70. O tamanho dos ROIs variou entre 36 pixels a 56 pixels.

Na FIGURA 3, é mostrada uma comparação de dados de reflectância de Mata de Galeria do PNCV, obtidas para sete datas diferentes, com os de reflectância padrão, obtidos no Distrito Federal, para as seis bandas do espectro refletivo do ETM+. Com base nesta figura, conclui-se que a conversão das sete cenas para reflectância foi relativamente satisfatória. Apesar dos valores de reflectância estarem, na maioria dos casos, superestimados, de uma forma geral, as sete curvas acompanharam o padrão espectral mostrado pela curva de referência.

Dados de curvas espectrais de vegetação verde disponíveis na literatura (MENESES \& NETTO, 2001) indicam valores de reflectância inferiores a 0,05 e 0,30 nas faixas espectrais do visível e do infravermelho próximo. Nas duas faixas espectrais do infravermelho médio, isto é, de 1,5 $\mathrm{mm}$ a $1,8 \mathrm{~mm}$ e de $2,1 \mathrm{~mm}$ a $2,4 \mathrm{~mm}$, as referidas reflectâncias raramente ultrapassam $0,15 \mathrm{~mm}$ e $0,05 \mathrm{~mm}$, respectivamente. De uma forma geral, as conversões efetuadas estão de acordo com esses dados de literatura. 
Identificação de Cerrado Rupestre por Meio de Imagens Multitemporais do Landsat: Proposta Metodológica Erika Regina Prado Nascimento, Edson Eyji Sano

As curvas espectrais acumuladas das reflectâncias das sete cenas para quatro coberturas de terras do parque (FIGURA 4; FIGURA5) indicam uma grande melhoria no potencial para discriminar Cerrado Rupestre. Percebe-se uma clara dificuldade em discriminar as quatro classes espectrais em qualquer uma das cenas individuais analisadas no período de junho a outubro (estação seca). No entanto, quando as respostas espectrais são acumuladas, o poder de discriminação dos alvos tende a aumentar. Quanto maior o número de cenas, maior é a capacidade de discriminação. Essa capacidade maior de separabilidade foi mais clara para as bandas 3, 4, 5 e 7. As confusões espectrais entre massas d'água e formações florestais na banda 3 e entre áreas agrícolas e formações florestais na banda 4 podem ser minimizadas nas outras bandas, notadamente, nas bandas 5 e 7 .

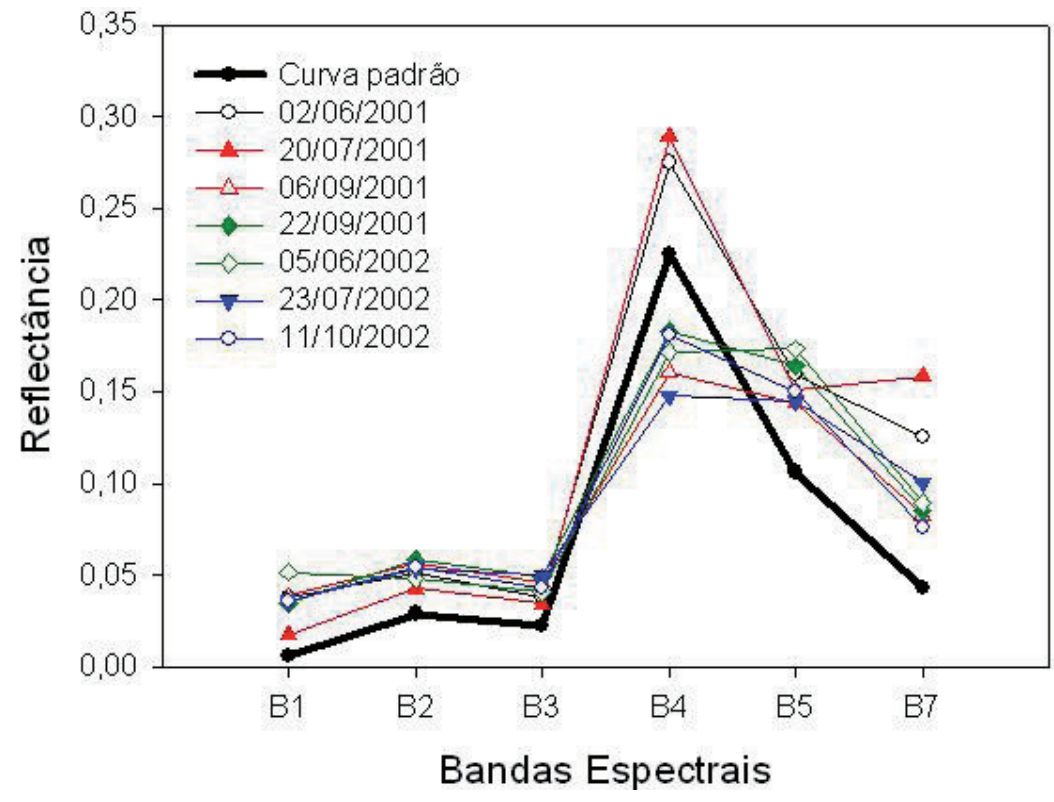

FIGURA 3: Comparação dos dados de reflectância referentes à Mata de Galeria das sete cenas que cobrem o Parque Nacional da Chapada dos Veadeiros com dados de referência do mesmo alvo. 


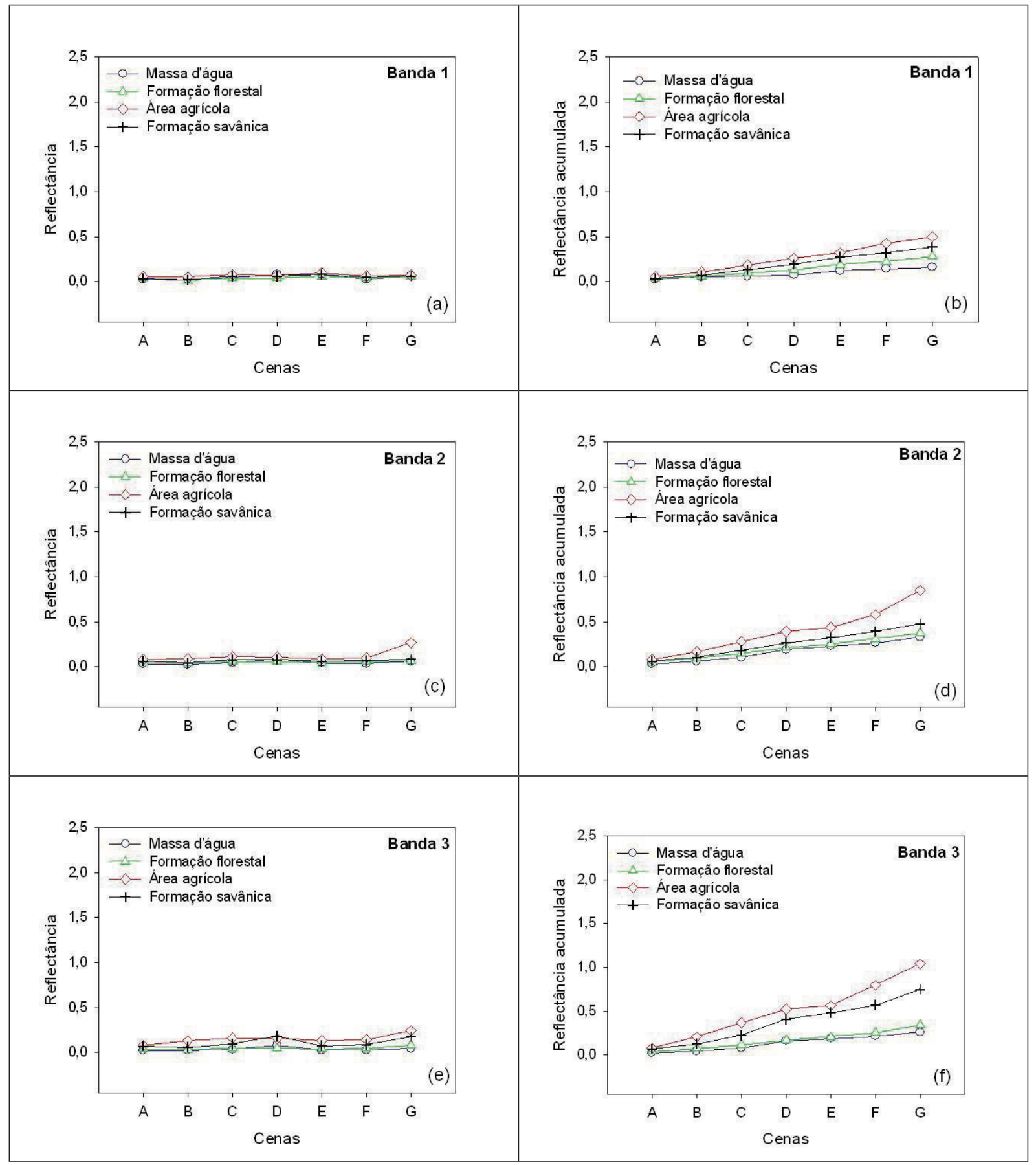

FIGURA 4: Reflectâncias individual e acumulada das bandas 1, 2 e 3 do Landsat ETM+ para sete cenas e quatro classes de cobertura de terras do Parque Nacional da Chapada dos Veadeiros e adjacências. Datas de passagem: A = 02/06/2001; $\mathrm{B}=20 / 07 / 2001 ; \mathrm{C}=06 / 09 / 2001 ; \mathrm{D}=22 / 09 / 2001 ; \mathrm{E}=05 / 06 / 2002 ; \mathrm{F}=23 / 07 / 2002 ; \mathrm{e} \mathrm{G}=11 / 10 / 2002$. 
Identificação de Cerrado Rupestre por Meio de Imagens Multitemporais do Landsat: Proposta Metodológica Erika Regina Prado Nascimento, Edson Eyji Sano

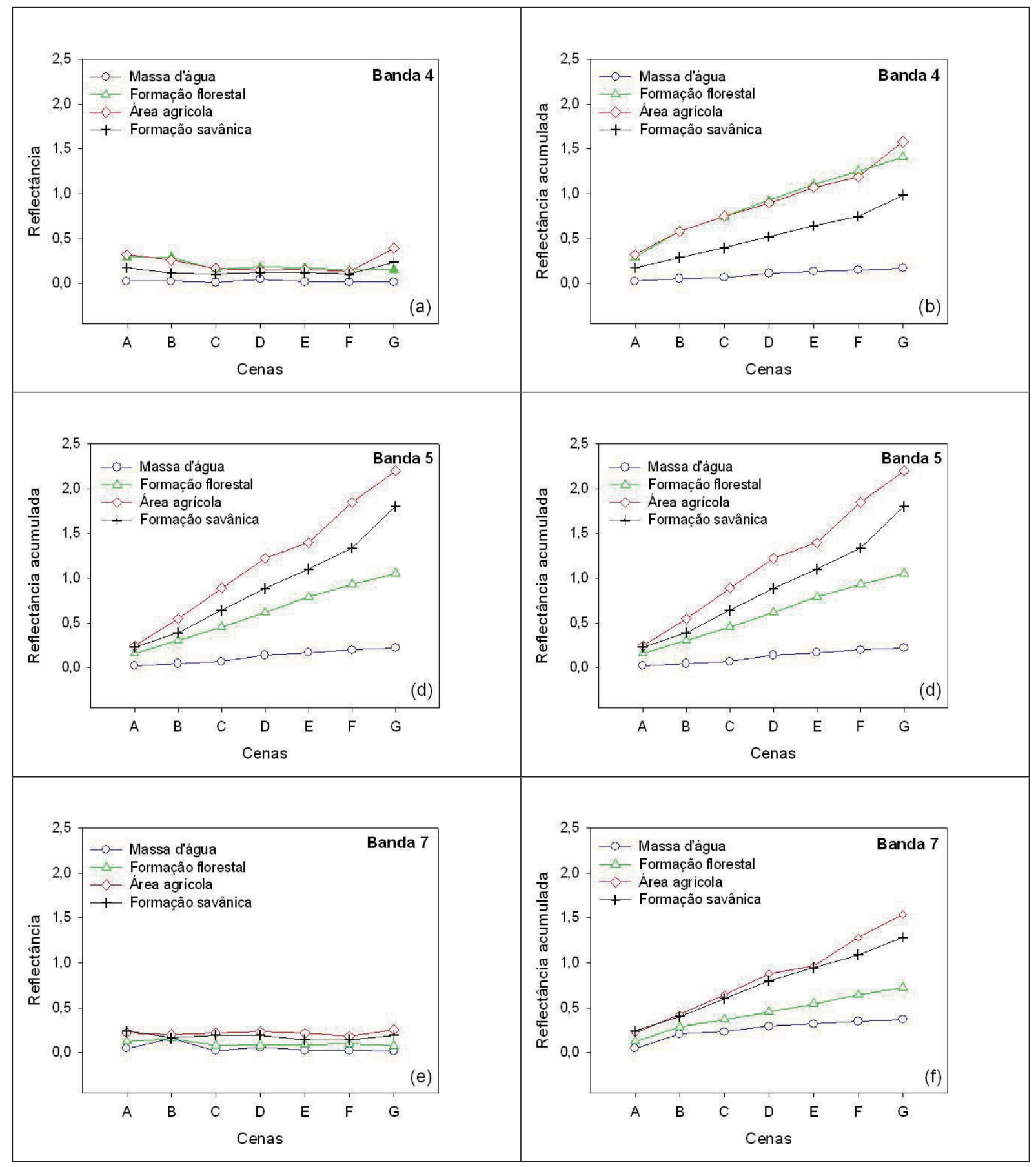

FIGURA 5: Reflectâncias individual e acumulada das bandas 4, 5 e 7 do Landsat ETM+ para sete cenas e quatro classes de cobertura de terras do Parque Nacional da Chapada dos Veadeiros e adjacências. Datas de passagem: A = 02/06/2001; $\mathrm{B}=20 / 07 / 2001 ; \mathrm{C}=06 / 09 / 2001 ; \mathrm{D}=22 / 09 / 2001 ; \mathrm{E}=05 / 06 / 2002 ; \mathrm{F}=23 / 07 / 2002 ;$ e $\mathrm{G}=11 / 10 / 2002$. 
Esses resultados estão de acordo com os obtidos por Ratana et al. (2005) que analisaram índices de vegetação (NDVI, índice de vegetação normalizada pela diferença e EVI, índice de vegetação realçado) derivados do sensor MODIS e acumulados para o período de janeiro a dezembro. De acordo com esses autores, as formações campestres e savânicas do Cerrado tendem a apresentar um perfil sigmoidal devido à elevada sensibilidade dessas formações à sazonalidade climática. As formações florestais tendem a apresentar curvas mais retilíneas e com índices de vegetação mais altos.

Foram mapeados 24.451 hectares de Cerrado Rupestre no PNCV, o que corresponde a $37 \%$ da área do parque (FIGURA 6). Tais áreas encontramse distribuídas ao longo de toda a área de estudo, notadamente ao longo de um eixo longitudinal NE - SW que passa aproximadamente pelo centro do parque.

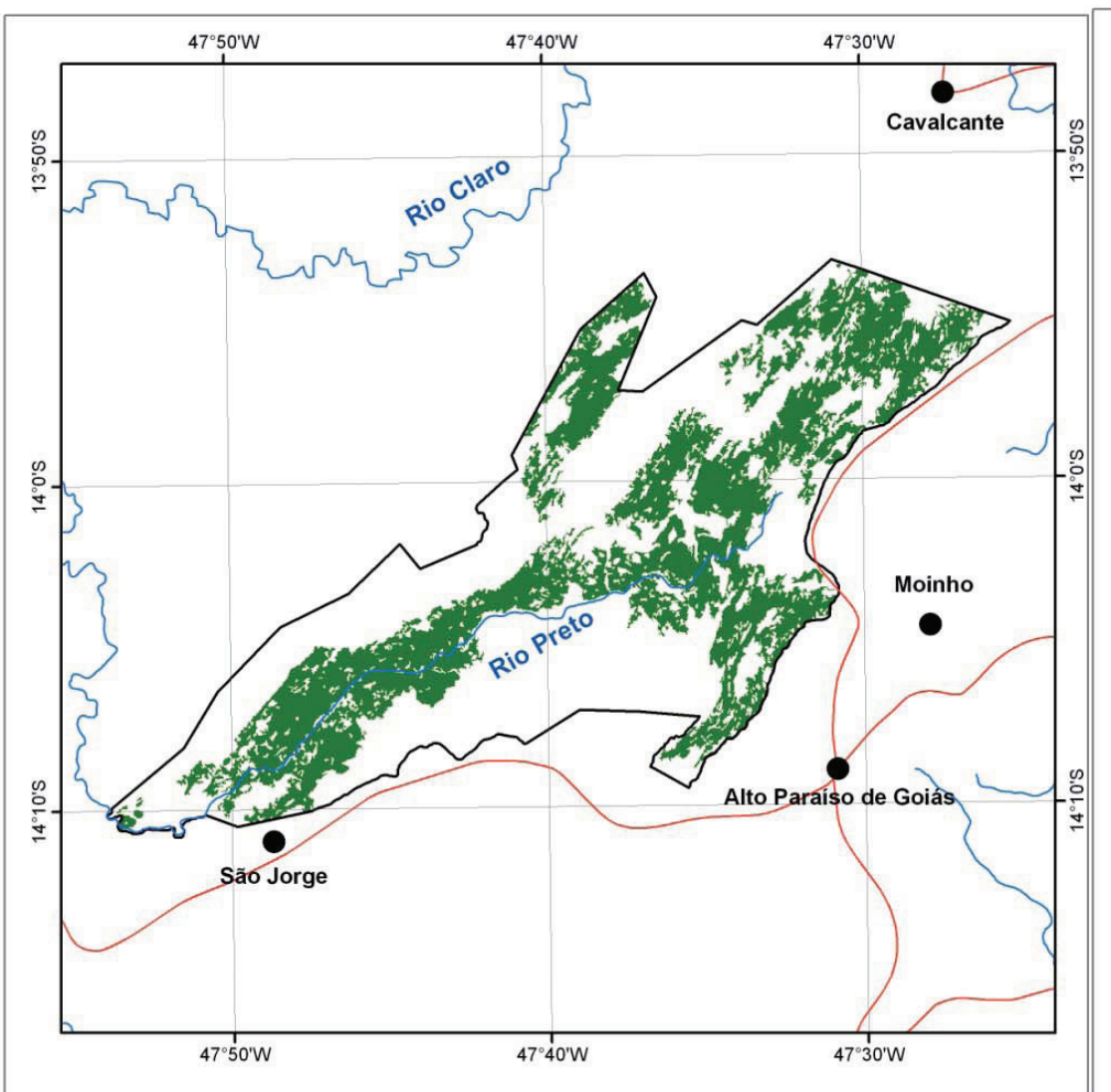

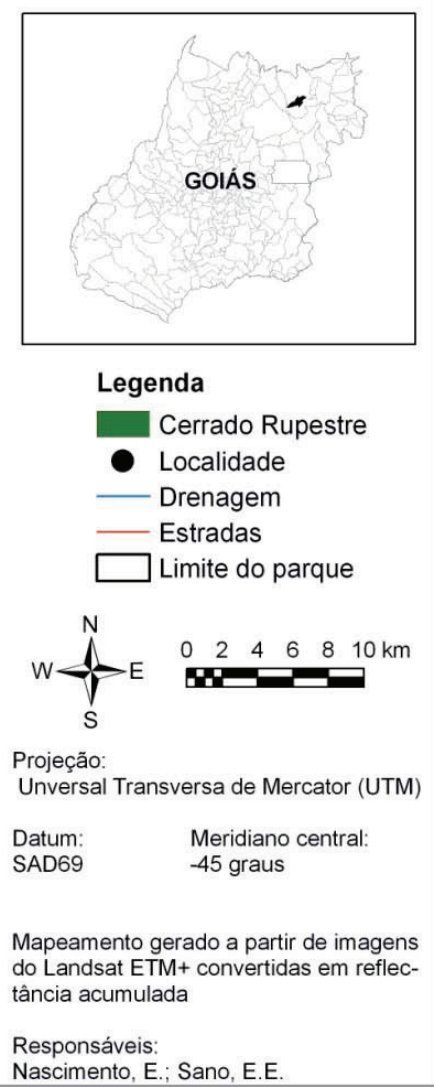

Nascimento, E.; Sano, E.E.

FIGURA 6: Mapa de ocorrência de Cerrado Rupestre no Parque Nacional da Chapada dos Veadeiros, elaborado a partir de interpretação visual de imagens do Landsat ETM+ convertida em reflectância acumulada.

O índice de exatidão global do mapeamento das áreas de Cerrado Rupestre no PNCV foi de $85 \%$ (TABELA2). De um total de 699 pontos identificados no mosaico do Ikonos e QuickBird, 594 pontos foram mapeados corretamente. Não houve tendência do mapeamento em apresentar maior erro de omissão ou de comissão: ambos os erros ficaram em 17\%.
São considerados erros de omissão, aqueles pontos que realmente correspondem a Cerrado Rupestre e que foram mapeados como "Não-Rupestre". São considerados erros de comissão, aqueles pontos que não correspondem a Cerrado Rupestre e que foram mapeados como tal. 
Identificação de Cerrado Rupestre por Meio de Imagens Multitemporais do Landsat: Proposta Metodológica Erika Regina Prado Nascimento, Edson Eyji Sano

TABELA 2: Análise da exatidão global e erros de omissão e comissão do mapa de ocorrência de Cerrado Rupestre no Parque Nacional da Chapada dos Veadeiros. A verdade terrestre foi obtida a partir da análise visual de mosaico de imagens do Ikonos e QuickBird.

\begin{tabular}{|c|c|c|c|c|}
\hline \multirow{6}{*}{ 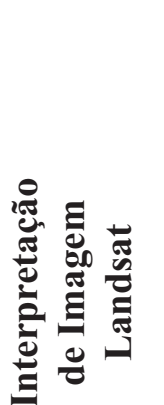 } & \multicolumn{4}{|c|}{ Verdade Terrestre } \\
\hline & & Cerrado Rupestre & $\begin{array}{l}\text { Cerrado Não- } \\
\text { Rupestre }\end{array}$ & SUBTOTAL \\
\hline & Cerrado & & & \\
\hline & Rupestre & 261 & 59 & 320 \\
\hline & Cerrado & & & \\
\hline & Não-Rupestre & 46 & 333 & 379 \\
\hline & SUBTOTAL & 307 & 392 & 699 \\
\hline
\end{tabular}

De acordo com Lima (2008), em termos espectrais, Cerrado Rupestre confunde principalmente com a Mata Seca e o Cerrado sentido restrito. Um outro fator que contribui para a diminuição da exatidão global é a transição gradual e complexa do Cerrado Rupestre para as fisionomias adjacentes (OLIVEIRA-FILHO \& FLUMINHAN-FILHO, 1999). De acordo com Ribeiro \& Walter (2008), o Cerrado Rupestre ocorre em ilhas mescladas às outras fitofisionomias de formação savânica. Numa toposequência, é comum encontrarem Cerrado Rupestre nas porções mais elevadas e Mata Seca ou Cerrado sentido restrito nas porções mais intermediárias e baixas, dificultando a discriminação de Cerrado Rupestre nas imagens Landsat (LIMA, 2008).

\section{CONSIDERAÇÕES FINAIS}

A análise visual de imagens multitemporais do Landsat ETM+ convertidas para reflectância acumulada e processadas pela técnica de segmentação de imagens por crescimento de regiões permitiu uma boa discriminação de ocorrências de Cerrado Rupestre do Parque Nacional da Chapada dos Veadeiros. Essa abordagem metodológica pode ser estendida para outras fitofisionomias ou outras coberturas de terras ou mesmo para outras regiões geográficas. A estratégia metodológica proposta nesse estudo pode ser ainda considerada promissora por causa da relativamente recente disponibilização sem custos de todas as imagens da série Landsat do território brasileiro disponíveis no arquivo do Inpe.

A técnica de correção atmosférica pelo FLAASH mostrou ser eficiente na conversão de valores de radiância do topo da atmosfera das imagens do Landsat ETM+ para reflectância na superfície terrestre, produzindo séries temporais relativamente homogêneas do Landsat, em termos de interferência atmosférica. No entanto, a referida conversão não funcionou a contento para imagens do Landsat TM.

Os dois principais fatores negativos que podem ter prejudicado um melhor desempenho do mapeamento final foi a impossibilidade de inclusão de imagens do sensor TM e a indisponibilidade de imagens ETM+ sem cobertura de nuvens da estação chuvosa na área de estudo.

Em termos de futuras pesquisas, recomendase a inclusão da técnica de correção topográfica em adição à correção atmosférica (ortorretificação). Embora raramente empregados nos protocolos de processamento multitemporal de imagens Landsat, os requerimentos para a referida correção são relativamente simples, exigindo-se apenas o uso de modelos digitais de elevação na mesma resolução espacial das imagens Landsat (VICENTESERRANO et al., 2008). Esses autores encontraram diferenças bastante perceptíveis na evolução 

Erika Regina Prado Nascimento, Edson Eyji Sano

temporal de dados de reflectância de superfície de terrenos com faces orientadas para norte e para sul, decorrentes das diferenças nas condições de iluminação solar. Uma outra técnica recomendada é o da normalização radiométrica relativa, em que uma determinada cena (frequentemente, a mais recente ou aquela com menor interferência atmosférica, livres de cobertura de nuvens) é definida como referência. As outras cenas são então normalizadas em relação a essa cena de referência. Estudos anteriores como os desenvolvidos, por exemplo, por Schroeder et al. (2006) indicaram que a correção atmosférica per si so é incapaz de remover ruídos extra-superfície de series temporais.

Considerando ainda que o Cerrado Rupestre ocorre em áreas de relevo movimentado, formado por solos classificados em Neossolos Litólicos é interessante utilizar em trabalhos futuros informações topográficas que corroborem na delimitação de áreas de Cerrado Rupestre.

\section{REFERÊNCIAS}

BRITES, R.S.; SOARES, V.P.; RIBEIRO, C.A.A.S. Verificação da exatidão em classificações de uma imagem orbital mediante a utilização de três índices. Árvore, v. 20, p. 415 - 424, 1996.

CAMPBELL, J.B. Introduction to Remote Sensing. Nova Iorque: The Guilford Press, 4. ed., 2007.

CERQUEIRA, J.D.; CANDEIAS, A.L.B.; SILVA, D.C. Orientação de imagens Ikonos utilizando a transformação matemática APM - Affine Projection Model. In: CONGRESSO BRASILEIRO DE CARTOGRAFIA, 21, Belo Horizonte, 2003. Anais... Disponível em: < http://www.cartografia.org.br/xxi cbc/201-SR22.pdf>. Acesso em: 20 nov. 2009.

GEOAMBIENTE. Projeto JICA (Corredor ecológico do Cerrado Paranã - Pirineus). Metodologia empregada no processamento das imagens QuickBird, Ikonos e Aster. São José dos Campos, 2005. (relatório técnico não publicado).
JENSEN, J.R. Introductory Digital Image Processing: A Remote Sensing Perspective. Upper Saddle River: Prentice Hall, 2. ed., 1996.

JUSTICE, C.; BELWARD, A.; MORISETTE, J.; LEWIS, P.; PRIVETTE, J.; BARET, F. Developments in the 'validation' of satellite sensor products for the study of land surface. International Journal of Remote Sensing, vol. 21, p. 3383-3390, 2000.

LIESENBERG, V.; SOARES, L.G.; PONZONI, F.J. Variations in reflectance with seasonality and viewing geometry: implications for classification of Brazilian savanna physiognomies with MISR/Terra data. Remote Sensing of Environment, vol. 107, p. 276 - 286, 2007.

LIMA, C. A. O Cerrado Rupestre no Estado de Goiás com base em imagens LANDSAT ETM+. 2008, 116f. Dissertação (Mestrado em Engenharia Florestal) - Universidade de Brasília, Departamento de Engenharia Florestal, Brasília, 2008.

MENESES, P.R.; NETTO, J.S.M. Sensoriamento Remoto: reflectância dos alvos naturais. Brasília: Editora UnB, 1 1 ed., 2001.

MORISETTE, J.T.; NICKESON, J.E.; DAVIS, P.; WANG, Y.; TIAN, Y.; WOODCOCK, C.E.; SHABANOV, N.; HANSEN, M.; COHEN, W.B.; OETTER, D.R.; KENNEDY, R.E. High spatial resolution satellite observations for validation of MODIS land products: IKONOS observations acquired under the NASA scientific data purchase. Remote Sensing of Environment, vol. 88, p. $100-$ 110, 2003.

OLIVEIRA-FILHO, A.T.; FLUMINHAN-FILHO, M.. Ecologia da vegetação do Parque Florestal Queda do Rio Bonito. Cerne, vol. 5, p. 51-64, 1999.

RATANA, P.; HUETE, A.; FERREIRA, L.G. Analysis of Cerrado physiognomies and conversion in the MODIS seasonal-temporal domain. Earth Interactions, v. 9, p. $1-22,2005$. 
Identificação de Cerrado Rupestre por Meio de Imagens Multitemporais do Landsat: Proposta Metodológica Erika Regina Prado Nascimento, Edson Eyji Sano

REATTO, A.; CORREIA, J.R.; SPERA, S.T.; MARTINS, E.S. Solos do bioma Cerrado: aspectos pedológicos. In: SANO, S.M.; ALMEIDA, S.P.; RIBEIRO, J.F. (eds.), Cerrado. Ecologia e Flora. Planaltina, Embrapa Cerrados, v. 1, Cap. 5, p. 107 $-134$.

RIBEIRO, J. F.; WALTER, B.M.T. 2008. As principais fitofisionomias do Cerrado. In: SANO, S.M.; ALMEIDA, S.P.; RIBEIRO, J.F. (eds.), Cerrado. Ecologia e Flora. Planaltina, Embrapa Cerrados, v. 1, Cap. 6, p. $151-199,2008$.

SANO, E.E.; FERREIRA, L.G.; HUETE, A.R. Synthetic aperture radar (L-band) and optical vegetation indices for discriminating the Brazilian savanna physiognomies: a comparative analysis. Earth Interactions, vol. 9, Paper n. 15, p. 1-15, 2005.

SANO, E.E.; FERREIRA, L.G.; ASNER, G.P.; STEINKE, E.T. Spatial and temporal probabilities of obtaining cloud-free Landsat images over the Brazilian tropical savanna. International Journal of Remote Sensing, vol. 28, n. 12, p. 2739-2752, 2007.

SCHOWENGERDT, R.A. Remote Sensing. Models and Methods for Image Processing. San Diego: Academic Press, 3. ed., 2007.

VICENTE-SERRANO S.M.; PEREZ-CABELLO F.; LASANTA T. Assessment of radiometric correction techniques in analyzing vegetation variability and change using time series of Landsat images. Remote Sensing of Environment, v.112, p. 3916 -3934, 2008. 